\title{
Ground-based astrometry calibrated by Gaia DR1: new perspectives in asteroid orbit determination
}

\author{
F. Spoto ${ }^{1, \star}$, P. Tanga ${ }^{1}$, S. Bouquillon ${ }^{2}$, J. Desmars ${ }^{3}$, D. Hestroffer ${ }^{3}$, F. Mignard ${ }^{1}$, M. Altmann ${ }^{2,4}$, D. Herald ${ }^{5}$, \\ J. Marchant ${ }^{6}$, C. Barache ${ }^{2}$, T. Carlucci ${ }^{2}$, T. Lister ${ }^{7}$, and F. Taris ${ }^{2}$ \\ ${ }^{1}$ Université Côte d'Azur, Observatoire de la Côte d'Azur, CNRS, Laboratoire Lagrange, route de l'Observatoire, \\ 06304 Nice Cedex 4, France \\ e-mail: fspoto@oca.eu \\ 2 SYRTE, Observatoire de Paris, PSL Research University, CNRS, Sorbonne Universités, UPMC Univ. Paris 06, \\ 61 avenue de l'Observatoire, 75014 Paris, France \\ 3 IMCCE, Observatoire de Paris, PSL Research University, CNRS, Sorbonne Universités, UPMC Univ. Paris 06, Univ. Lille, \\ 77 av. Denfert-Rochereau 75014 Paris, France \\ ${ }^{4}$ Zentrum für Astronomie der Universität Heidelberg, ARI, 69120 Heidelberg, Germany \\ 5 International Occultation Timing Association (IOTA), Observatoire de la Côte d'Azur, Bv de l'Observatoire, CS 34229, \\ 06304 Nice Cedex 4, France \\ ${ }^{6}$ Liverpool John Moores University, ARI, Liverpool, L3 5UA, UK \\ 7 Las Cumbres Observatory, Goleta, CA 93117, USA
}

Received 24 June 2017 / Accepted 13 August 2017

\begin{abstract}
Context. The Gaia Data Release 1 (GDR1) is a first, important step on the path of evolution of astrometric accuracy towards a much improved situation. Although asteroids are not present in GDR1, this intermediate release already impacts asteroid astrometry.

Aims. Our goal is to investigate how the GDR1 can change the approach to a few typical problems, including the determination of orbits from short-arc astrometry, the exploitation of stellar occultations, and the impact risk assessment.

Methods. We employ optimised asteroid orbit determination tools, and study the resulting orbit accuracy and post-fit residuals. For this goal, we use selected ground-based asteroid astrometry, and occultation events observed in the past. All measurements are calibrated by using GDR1 stars.

Results. We show that, by adopting GDR1, very short measurement arcs can already provide interesting orbital solutions, capable of correctly identifying near-Earth asteroids (NEAs) and providing a much more accurate risk rating. We also demonstrate that occultations, previously used to derive asteroid size and shapes, now reach a new level of accuracy at which they can be fruitfully used to obtain astrometry at the level of accuracy of Gaia star positions.
\end{abstract}

Key words. astrometry - occultations - minor planets, asteroids: general - catalogs - celestial mechanics

\section{Introduction}

The ESA Gaia collaboration has published the first Gaia data release (GDR1) containing different data sets of stellar astrometry (Gaia Collaboration 2016). Although it is based on a first, preliminary calibration to be improved by future releases, GDR1 already represents a huge jump in our knowledge of the sky (Lindegren et al. 2016). Roughly quantified as a factor $\sim 10$ in accuracy, this improvement opens immediate perspectives of scientific exploitation in many branches of astrophysics.

Gaia is also directly measuring asteroid positions, but they are not yet present in GDR1 and will appear in the future intermediate releases (starting with GDR2, April 2018).

While direct measurements of asteroids by Gaia will provide the most accurate positions available (Tanga et al. 2008), the improvement in stellar astrometry itself can potentially change all other approaches where stars are used as astrometric reference.

In this article, we present the first results obtained in this respect, by exploiting both recent images, optimally calibrated

^ F. Spoto acknowledges support by the CNES post-doctoral program. and reduced by GDR1, and stellar occultations whose target star is contained in GDR1.

Our motivation is twofold:

- First, Gaia provides an all-sky, dense system of astrometric reference sources, that can be used to measure all asteroids, even those that are beyond reach for Gaia due to flux limit or geometric configuration.

- Second, this approach extends in time - beyond the Gaia mission duration - the possibility of measuring asteroid positions at very high accuracy by applying a better calibration to old and new data.

Although the intermediate accuracy of GDR1 is going to be surpassed by forthcoming releases, the indications that we obtain in this evolving context are already significant and clearly indicate the change of paradigm brought by Gaia. For our application, the most relevant information to come will be parallaxes and proper motions for the whole sample of $\sim 1$ billion stars, that will be made available in GDR2. Already in GDR1 however, the Tycho-Gaia Astrometric Solution (TGAS) provides these parameters at very high accuracy for $\sim 2$ million stars, exploiting 
the long time base between Gaia and its pioneering precursor, HIPPARCOS/Tycho.

Desmars et al. (2013) have studied the current situation of orbital uncertainties for asteroids. Their statistics on the content of the Minor Planet Center (MPC) observation database show that most of the observations are Charge-Coupled Device (CCD) measurements with an average accuracy of $\sim 400$ mas. Optimised surveys can reach much better accuracy levels, but limitations due to zonal and systematic errors of the catalogues prevent us from reaching uncertainties better than $\sim 40$ mas (Farnocchia et al. 2015a).

Past studies, such as Chesley et al. (2010), Farnocchia et al. (2015a), have analysed different catalogues to derive local corrections that can be used to improve the available set of astrometric measurements archived at the MPC. This approach can be applied to the whole record of the existing astrometry, while the calibration of the raw data (CCD images, in general) is not re-processed. On the other hand, our goal hereinafter is to assess the impact of GDR1 at the very beginning of the process, that is starting from the calibration procedures.

We consider two data sources that are expected to provide very precise data. The first one is the CCD astrometry provided by the Ground-Based Optical Tracking (GBOT) of Gaia. This activity, which runs on several telescopes, regularly obtains astrometric data of the Gaia satellite itself since its launch in Dec. 2013. As the Gaia trajectory sweeps a relatively large sky area around opposition, in the direction of L2, GBOT observes and discovers asteroids (typically between 10 and 80 each night, of which $\sim 46 \%$ are new discoveries). Starting with the availability of GDR1 in September 2016, GBOT has exploited it for the calibration of the astrometry. Asteroid positions are regularly submitted to the MPC.

The second data source is provided by the data set of stellar occultations by asteroids, observed in the past. Such events can provide very accurate positions of the asteroid relative to the occulted star. The best results are obtained when the sky-projected shape of the asteroid is sampled by several occultation chords.

We describe in detail our approach in the following sections. GBOT calibration of asteroid astrometry is illustrated in Sect. 2. We investigate the evolution of the impact rating for the first confirmed near-Earth asteroid (NEA) discovered by GBOT, using GDR1 in Sect. 3. The improvement of short-arc orbit determination is then studied in Sect. 4. Section 5 is devoted to the exploitation of the stellar occultations. Finally our results are summarised in Sect. 6.

\section{Astrometric calibration of Ground Based Optical Tracking data by Gaia DR1}

GBOT is an observation campaign to organise and carry out the high- precision astrometric tracking of the Gaia satellite itself, in the frame of the Data Processing and Analysis Consortium (DPAC) of Gaia.

Its goal is to fully ensure the elimination of systematic effects, for example, aberration, even for those objects which can be measured to the greatest precision (Altmann et al. 2014) and for which traditional radar tracking methods alone are not fully sufficient.

The GBOT approach is based on daily CCD observations performed throughout the mission. Currently GBOT mainly uses two telescopes: the VLT Survey Telescope (VST) installed at ESO's Paranal Observatory in Chile and the Liverpool Telescope (LT) on La Palma (Canary Islands, Spain). Each night, each telescope attempts to take a sequence of frames on which the Gaia satellite itself is seen as a faint and fast moving object (its magnitude is about $R \sim 21$ and its sidereal speed can reach $40 \mathrm{mas} / \mathrm{s}$ ).

The Gaia mission requirement for the absolute accuracy on the satellite position determination in the International Celestial Reference Frame (ICRF) is $150 \mathrm{~m}$, which translates to 20 mas in the plane of sky. This precision cannot be reached by the usual radar ranging and communications stations, which can only deliver $\sim 2000 \mathrm{~m}$ in position and $10 \mathrm{~mm} \mathrm{~s}^{-1}$ in velocity on the sky. A specific tool - the GBOT Astrometric Reduction pipeline (GARP) -has been developed to reach this level of precision and accuracy for the reduction of images of moving objects - that is, trailed images on the CCD frame (Bouquillon et al. 2014). Note that since the VST tracking is locked on the Gaia speed, the satellite is recorded as a stationary object while the stars are recorded as trailed, elongated, images.

Since the beginning of 2015 , the GBOT group decided to also measure with the help of GARP the solar system object (SSO) present in the one square degree field of VST and in the $10^{\prime} \times 10^{\prime}$ field of LT. In April 2017, more than 12000 asteroid positions were recorded and submitted to the MPC.

GARP proceeds, first, by determining the photo-centre position of the object as if it was non-moving. The drift angle and amplitude are determined from the ICRF positions in the first and last frames of the whole sequence. In a second iteration, a linearly moving Gaussian to the drifting PSF is fitted (Bouquillon et al. 2017).

The precision of this photo-centre determination is the result of complicated interactions between the brightness of the object, the elongation due to the drift and the conditions of observation (seeing, pixel size, background flux, etc.; see Bouquillon et al. 2017 , for more details). For a bright and slowly moving main belt asteroid such as (1132) Hollandia $(R \sim 14.5 \mathrm{mag}, S / N \sim 800$, elongation $\sim 2$ Pixels), which is one of the brighter MBAs found in the GBOT data, the uncertainty is around 5 mas for an exposure time of one minute. For a faint and fast object observed in similar conditions, such as the NEA $2016 E K_{85}(R \sim 20 \mathrm{mag}$, $S / N \sim 10$, elongation $\sim 21$ Pixels), the centroiding error can reach 100 mas of which the largest part is along the drift.

For the astrometric calibration, we use a reference catalogue as a realisation of the ICRF. For each image, we compute the standard coordinates of all reference stars potentially in the field of view by taking into account the aberration, the tropospheric effect and by applying a gnomonic projection. Then an algorithm based on the planar triangle method (Liebe 1995) makes the connection between each reference star and its counterpart in the image. Finally, two polynomials are fitted to perform the link between the $X$ and $Y$ coordinates of the CCD frame and the standard coordinates. For OMEGACAM at VST a polynomial of first degree is sufficient, while three degrees are necessary for the IO:O camera at LT.

Using Gaia DR1 (Gaia Collaboration 2016) as the reference catalogue, the standard deviation of the residuals between the catalogue and the data for all stars with $V<20.5$ mag is $\sim 30$ mas. This level of precision is not attainable without using Gaia astrometry as a reference catalogue. For instance, if we follow the same procedure of calibration with the PPMXL stars catalogue (Roeser et al. 2010), the standard deviation is 300 mas, that is ten times larger (see Fig. 1).

A second advantage of using Gaia DR1 as a reference catalogue, is to eliminate zonal errors. To illustrate this point, Fig. 1 presents the results of the GARP reductions of 21 sets of observations of the Gaia satellite itself, covering 13 consecutive nights in February 2016. Note that since the angular speed of Gaia is one degree per day, the fields of view of two sets of observations 

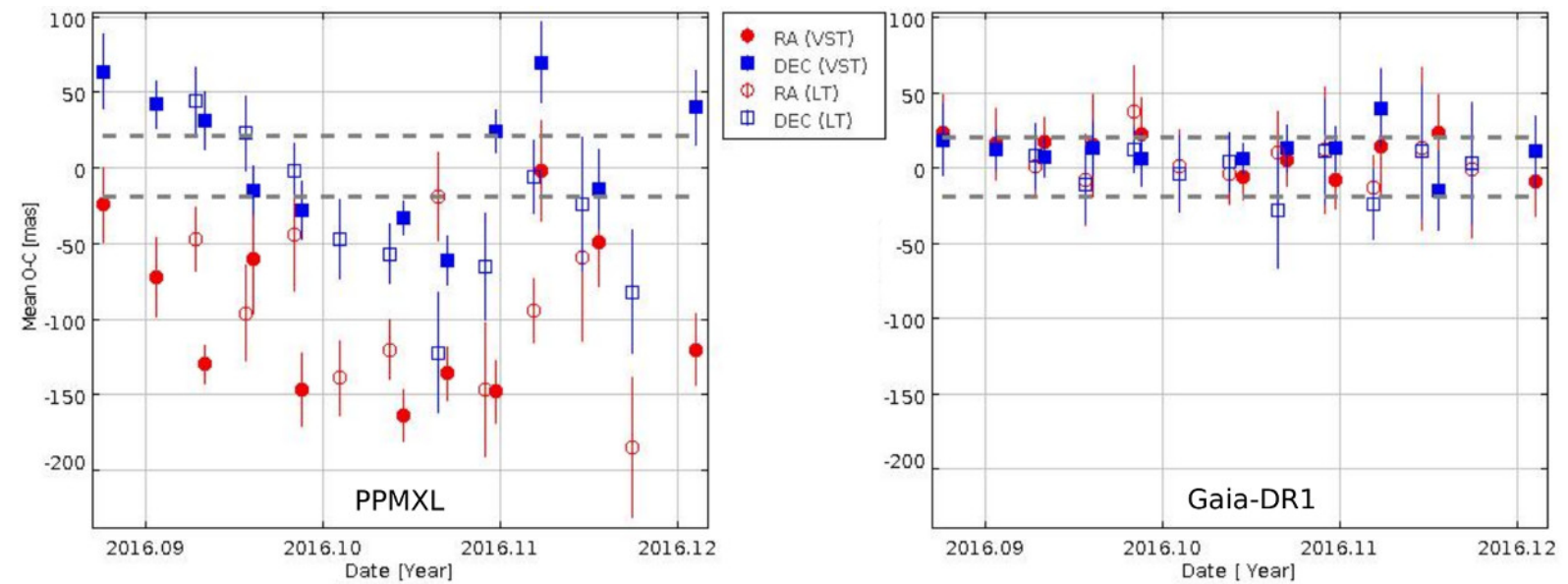

Fig. 1. Residuals between predicted and measured positions of the Gaia satellite. The astrometry has been calibrated with PPMXL (left panel) and Gaia DR1 (right panel). Each symbol corresponds to the average over a sequence of several frames over 30 min maximum (VST: filled symbols; LT: open symbols). Squares and circles indicate residuals in right ascension and declination respectively.

taken at a $24 \mathrm{~h}$ interval are completely different. In the case of the reduction with PPMXL (left panel), we observe a progressive variation of the daily mean differences between the Gaia position measured on the CCD frames and its ephemeris. Over a period of 13 days, these variations reach 150 mas in right ascension and around 100 mas in declination. These amplitudes are several times larger than the precision (around 30 mas) of each mean measurement in the reference frame of the PPMXL catalogue. This is confirmed by the calibration with Gaia DR1 (right panel), showing residuals below 20 mas, in good agreement with the ephemeris.

\section{NEOs and risk rating}

The first confirmed NEA discovered by GBOT is the asteroid $2016 E K_{85}$. This object represents a nice example illustrating how the accuracy of the astrometric calibration can affect the interpretation of short-arc orbital solutions in the frame of NEA impact monitoring.

$2016 E K_{85}$ was observed for the first time the night of March 9, 2016, by VST. It was then re-observed the following night by LT, and other observers.

The MPC classified the newly discovered object as a NEA and published the corresponding Minor Planet Electronic Circu$\operatorname{lar}\left(\right.$ MPEC) ${ }^{1}$, with 48 observations (28 GBOT and 20 from other observers). After the discovery of a new NEA (and whenever new observations are added), both NEODyS in Pisa ${ }^{2}$ and Sentry at the $\mathrm{JPL}^{3}$ check the possibility of impacts with the Earth for $100 \mathrm{yr}$ in the future.

At that early stage after the discovery, it turned out that the object had predicted possible impacts with the Earth in 2102 and 2106, with low impact probability (IP, Table 1).

One week later, on March 16, new observations at Mauna Kea (published on MPEC 2016-F48 ${ }^{4}$ ) produced a better orbital solution, definitely ruling out the possibility of an impact.

When $2016 E K_{85}$ was observed in March 2016, GDR1 was not yet available. We thus decided to reprocess the whole set

\footnotetext{
1 http://WwW . minorplanetcenter . net/mpec/K16/K16EC2 . html

2 http://newton.dm.unipi.it/neodys2/

3 http://neo.jpl.nasa.gov/risks/

4 http://www.minorplanetcenter.net/mpec/K16/K16F48. html
}

Table 1. Date, impact probability and Palermo scale rating of possible impacts with the Earth for the NEA $2016 E K_{85}$.

\begin{tabular}{ccc}
\hline \hline Date & IP & PS \\
\hline $2102 / 02 / 22.296$ & $1.24 \times 10^{-8}$ & -8.54 \\
$2102 / 02 / 22.549$ & $5.57 \times 10^{-8}$ & -7.89 \\
$2106 / 02 / 22.042$ & $6.82 \times 10^{-8}$ & -7.82 \\
$2106 / 02 / 22.311$ & $4.62 \times 10^{-7}$ & -6.99 \\
$2106 / 02 / 22.529$ & $1.20 \times 10^{-6}$ & -6.57 \\
$2106 / 02 / 22.605$ & $3.19 \times 10^{-7}$ & -7.14 \\
$2106 / 02 / 22.635$ & $8.93 \times 10^{-6}$ & -5.70 \\
\hline
\end{tabular}

Notes. The impact table appeared in NEODyS on the night of March 11, 2016. It has been computed using 48 optical observations from March 9 to March 11 by the OrbFit software version 5.0. All these data are consistent with data published by the JPL.

of 28 GBOT observations covering two nights (2016/03/09 and 2016/03/10), by exploiting the calibration based on GDR1. The sample contains 8 positions from the VST and 20 from the LT. Then we add to these 28 observations, the other 20 observations to reproduce the same initial set.

A preliminary orbit was computed with the Gauss method. The final orbit is obtained after a weighted least squares fit with an outlier- rejection procedure (Carpino et al. 2003). In principle, orbital fitting would require a weighting scheme based on an independent assessment of the accuracy obtained on asteroids by the application of different catalogues (Farnocchia et al. 2015a). In the case of GDR1 we searched for the appropriate weights consistent with the post-fit residuals.

Figure 2 shows the final residuals, that are typical of what we expect in GBOT for very faint moving sources. Few observations are discarded by the rejection procedure.

We consider three different orbits, one (Orbit 1) obtained using the initial set of observations as submitted at the MPC, another (Orbit 2) with the same set of observations, but where GBOT observations are reduced using GDR1, and the final orbit (Final Orbit) obtained using all the observations available at the MPC. We have compared Orbit 1 and Orbit 2 to Final orbit using different metrics. The result is that Orbit 2 is closer to Final orbit than Orbit 1 . This is of course due to the quality of the 


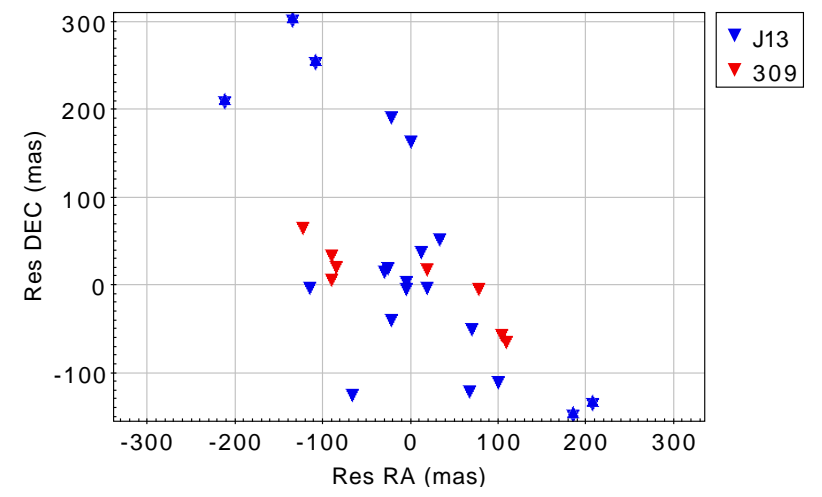

Fig. 2. Residuals in right ascension (RA) and declination (Dec) for the GBOT Gaia reduced observations. Stars represent the outliers discarded by the outlier-rejection procedure. The residuals are considered separately for the VST (MPC code 309) and the LT (MPC code J13).

Table 2. Orbit 1 and Orbit 2 compared to the final orbit available through two different metrics.

\begin{tabular}{ccc}
\hline \hline Orbits to be compared & $d$ & $d_{\mathrm{LoV}}$ \\
\hline Orbit 1 - Final orbit & 0.0010 & 1.20 \\
Orbit 2 - Final orbit & 0.0004 & 0.01 \\
\hline
\end{tabular}

Notes. $d$ is the difference in Equinoctial elements, and $d_{\mathrm{LoV}}$ is based on the identification algorithm applied to the Line of Variations (Milani et al. 2005b).

Gaia catalogue and to the consequent better reduction of GBOT observations.

We have used two different metrics to compare the orbits. The first one $(d)$ is the most simple, and it represents the difference of the Equinoctial orbital elements:

$d=\sqrt{\left(\frac{\left(a_{1}-a_{2}\right)}{\left(a_{1}+a_{2}\right)}\right)^{2}+\left(h_{1}-h_{2}\right)^{2}+\left(k_{1}-k_{2}\right)^{2}+\left(p_{1}-p_{2}\right)^{2}+\left(q_{1}-q_{2}\right)^{2}}$.

The second one $\left(d_{\mathrm{LoV}}\right)$ is based on the orbit identification algorithm, as described in Milani et al. (2005b). We use two sets of virtual asteroids (Milani et al. 2005a), and we compute the expected $\chi^{2}$ for the identification between each pair of virtual asteroids. Then we select the minimum value to obtain the final comparison. This identification algorithm is very useful when non-linearity plays an essential role, as in this case in which the time span by the observations in short. Table 2 summarises the results of the comparison, and shows that using both metrics Orbit 2 (obtained using GDR1) is always closer to the final orbit.

Based on the obtained orbit, we have looked for possible impacts with the Earth in the next $100 \mathrm{yr}$, as was done when the object was studied for the first time. We computed multiple solutions, and analysed each close encounter and each return that could lead to a possible impact. In this case, we do not find any possible impact risk. This result corresponds to our expectations, as a change in the astrometry due to an improved catalogue changes the direction of the Line of Variations (LoV, Milani et al. 2005a). This rules out all the possible impacts that were found with the preliminary solution based on a less precise catalogue.

Figure 3 shows the LoV using the same set of observations (GBOT): the left panel reproduces the situation as it was at the beginning when $2016 E K_{85}$ was put on the risk list, while the right panel shows what happens using observations reduced by GDR1. It is clear that in the first case the LoV pass through the
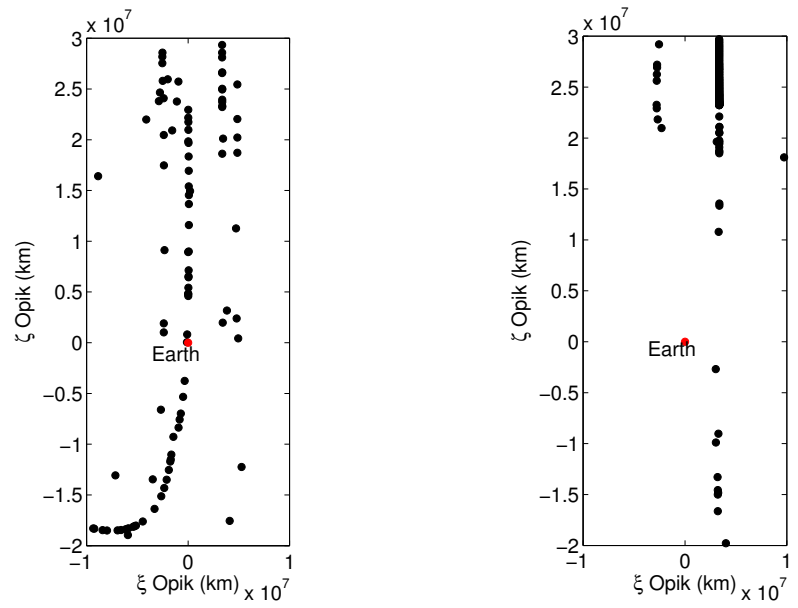

Fig. 3. Left: line of variations in the target plane of 2102 with GBOT observations as in the MPC observations file. Right: line of variations in the target plane of 2102 with GBOT observations reduced with the GDR1.

Earth, while in the second it is clearly displaced. The LoV behaviour is very similar during the close encounter of 2106 as well.

\section{Exploitation of short observational arcs}

Besides the case of $2016 E K_{85}$ we decided to analyse other NEAs or MBAs observed by GBOT, to explore a wide variety of situations: from objects with few observations covering a very short time span of the order of several minutes, to others observed over one or two nights.

When we have too few observations or too short time span we encounter the worst possible scenario for the orbit determination: it is not possible to compute a preliminary orbit, neither to apply a least squares fit.

We then apply the systematic ranging (Farnocchia et al. 2015b; Spoto et al. 2017). This technique allows us to scan the admissible region (Milani et al. 2004) using a grid in the plane defined by topocentric distance and topocentric velocity. The systematic ranging is used when the amount of information in the observed arc is too limited to compute a six parameter orbit, and the differential correction procedure fails. Our goal is thus simply to provide a first constraint on the family of orbits that are compatible with the astrometry, and try to distinguish NEAs from other categories.

As already pointed out in Sect. 3, the choice of the error model is crucial when we try to determine and fit an orbit, even when we apply the systematic ranging. Since we still do not have an error model that could represent the reduction with GDR1, we combine GBOT observations reduced with GDR1 with the whole set of ground-based observations available. We then fit the orbit and analyse GBOT residuals. For one case, namely (1132) Hollandia (already cited in Sect. 2), we have also compared the residuals obtained reducing GBOT observations with PPMXL and GDR1, respectively. Figure 4 shows the residuals of GBOT observations in right ascension and declination for the main belt (1132) Hollandia, reduced with PPMXL and GDR1 respectively. The reduction with the PPMXL catalogue clearly presents some zonal errors that are completely removed using the Gaia catalogue. 


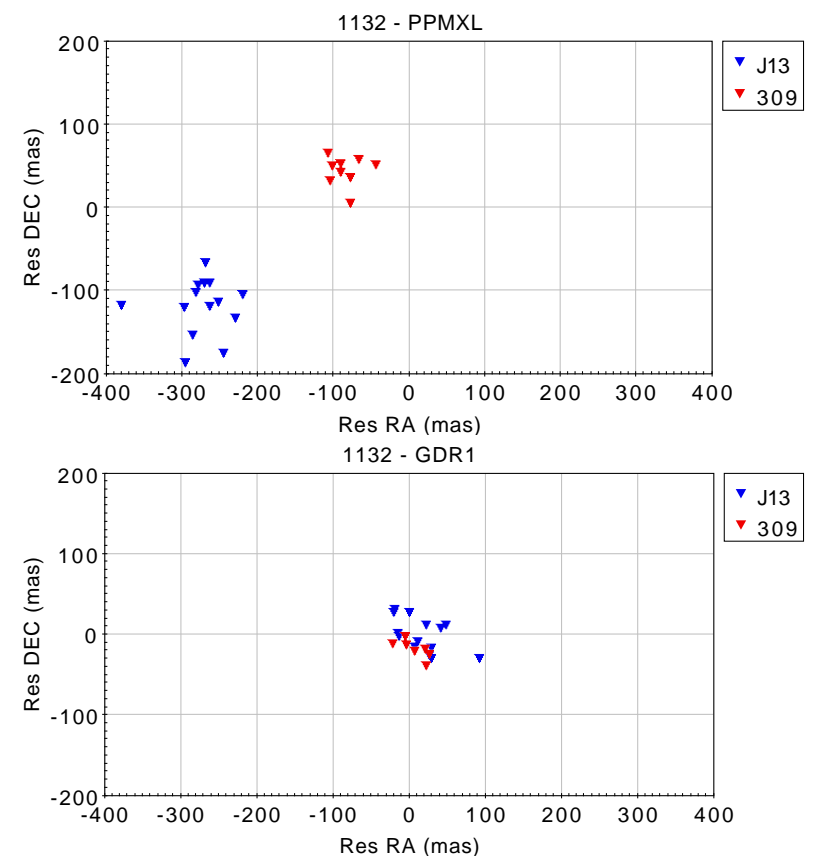

Fig. 4. Residuals in right ascension and declination for the main belt (1132) Hollandia of GBOT observations, reduced with PPMXL (upper panel) and GDR1 (bottom panel). In both cases, the residuals are considered separately for the VST (MPC code 309) and the LT (MPC code $J 13)$.

Table 3. Asteroid number, provisional designation, weights in right ascension (RA) and declination (Dec) for the two main GBOT observatories: LT (J13) and VST (309).

\begin{tabular}{rlrrrr}
\hline \hline Number & $\begin{array}{l}\text { Provisional } \\
\text { designation }\end{array}$ & $\begin{array}{r}\text { J13 RA } \\
\text { (mas) }\end{array}$ & $\begin{array}{r}\text { J13 Dec } \\
\text { (mas) }\end{array}$ & $\begin{array}{r}309 \text { RA } \\
\text { (mas) }\end{array}$ & $\begin{array}{r}309 \text { Dec } \\
\text { (mas) }\end{array}$ \\
\hline 18109 & $2000 N G_{11}$ & & & 60 & 50 \\
21565 & $1998 Q Z_{102}$ & 50 & 70 & 30 & 20 \\
32998 & $1997 \mathrm{CK}_{5}$ & 30 & 20 & 20 & 30 \\
79134 & $1990 V O_{8}$ & 30 & 35 & 32 & 37 \\
110520 & $2001 T L_{79}$ & 23 & 34 & 24 & 30 \\
119177 & $2001 Q N_{61}$ & 37 & 31 & 34 & 26 \\
135121 & $2001 Q O_{137}$ & 43 & 30 & 30 & 30 \\
139832 & $2001 R L_{35}$ & 43 & 20 & 25 & 23 \\
162000 & $1990 O S$ & & & 40 & 50 \\
186822 & $2004 F E_{31}$ & & & 40 & 40 \\
190788 & $2001 R T_{17}$ & & & 66 & 70 \\
307301 & $2002 Q G_{20}$ & 60 & 50 & 34 & 33 \\
392704 & $2012 A E_{1}$ & & & 100 & 100 \\
\hline
\end{tabular}

We have computed the mean and the standard deviation of the residuals for each object analysed (see Table 3), and then we have applied the systematic ranging.

For the situations that we analyse, only the astrometric reduction by GDR1 produces exploitable results. We tested our approach on a set of NEAs and MBAs. We expect, for NEAs, to consistently obtain a high probability of being a genuine NEA, and that there is no confusion between the two categories (i.e. one should not find a MBA with a high NEA score).

Tables 4 and 5 summarise our results. All the objects have a small number of observations, and the time span is very short, usually less than $20 \mathrm{~min}$ for NEAs and $4 \mathrm{~h}$ for MBAs. The results are perfectly consistent, and confirm that GDR1 allows us
Table 4. Near-Earth asteroid number, provisional designation, total number of observations, time span covered (minutes), and probability of being a NEA.

\begin{tabular}{rlrcr}
\hline \hline Number & $\begin{array}{l}\text { Provisional } \\
\text { designation }\end{array}$ & No. obs & $\begin{array}{c}\text { Time span } \\
(\mathrm{min})\end{array}$ & $\begin{array}{r}\text { Probability } \\
\text { to be NEA }\end{array}$ \\
\hline 18109 & $2000 N G_{11}$ & 8 & 16 & 82 \\
162000 & $1990 O S$ & 10 & 16 & 89 \\
186822 & $2004 F E_{31}$ & 10 & 16 & 86 \\
190788 & $2001 R T_{17}$ & 10 & 16 & 100 \\
392704 & $2012 A E_{1}$ & 6 & 14 & 90 \\
\hline
\end{tabular}

Table 5. Main belt asteroid number, provisional designation, total number of observations, time span covered (hours), and probability of being a MBA.

\begin{tabular}{rlrcr}
\hline \hline Number & $\begin{array}{l}\text { Provisional } \\
\text { designation }\end{array}$ & No. obs & $\begin{array}{c}\text { Time span } \\
\text { (h) }\end{array}$ & $\begin{array}{r}\text { Probability } \\
\text { to be MBA }\end{array}$ \\
\hline 21565 & $1998 Q Z_{102}$ & 30 & 3.7 & 96 \\
32998 & $1997 C K_{5}$ & 30 & 3.0 & 100 \\
79134 & $1990 \mathrm{VO}_{8}$ & 29 & 3.5 & 90 \\
110520 & $2001 \mathrm{TL}_{79}$ & 15 & 3.0 & 83 \\
119177 & $2001 Q N_{61}$ & 28 & 3.6 & 80 \\
135121 & $2001 Q O_{137}$ & 30 & 3.5 & 99 \\
139832 & $2001 R L_{35}$ & 30 & 3.4 & 95 \\
307301 & $2002 Q G_{20}$ & 30 & 3.3 & 100 \\
\hline
\end{tabular}

to classify asteroid orbits, and identify new NEAs by exploiting ground-based astrometry on a very short observational arc.

\section{Astrometry by stellar occultations}

Improved orbits obtained by direct asteroid astrometry by Gaia, and improved stellar positions, are expected to strongly expand the number of stellar occultation predictions that have a good probability of success (Tanga \& Delbo 2007). This will provide obvious benefits for our capability to determine precise asteroid sizes. Also, asteroid shapes and satellite systems will be better constrained by a large amount of successful occultations.

Besides this promising perspective, we cannot neglect the value that the current record of positive stellar occultations has. In fact, stars contained in GDR1 that have been occulted by asteroids in the past correspond to very precise astrometric positions at the corresponding occultation epoch.

A complete list of occultation results, maintained by Dave Herald, is at the base of an available data set of observed events (Dunham et al. 2016). Most event epochs are after the year 2000, but some events have been observed starting from the late $70 \mathrm{~s}$. The earliest isolated occultation observed dates back to 1961 , for the asteroid (2) Pallas. The database above contains identifications of the occulted star, of the occulting asteroid, and the occultation parameters derived from the observations. Such parameters are computed for the geocentre, and include the apparent distance between the star and the asteroid at the minimum separation, and the epoch at which the minimum separation occurs. Both quantities are listed with their uncertainties, that we discuss in the following section.

\subsection{Accuracy budget}

The occultation accuracy can approach that of GDR1 for the stars, that is one or two orders of magnitude better than 


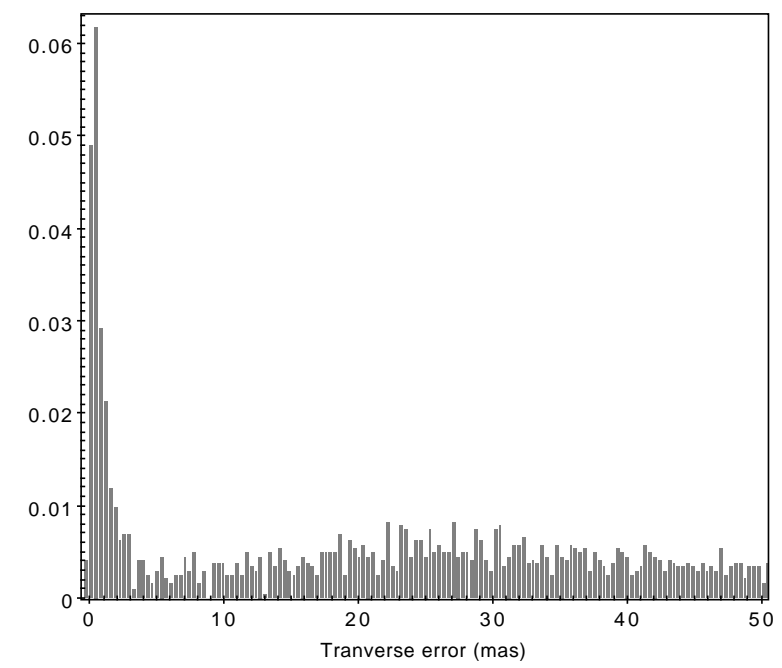

Fig. 5. Histogram of the astrometric error on the asteroid position in the direction perpendicular to the apparent motion of the asteroid during the occultation event.

traditional small-field imaging astrometry of asteroids from the ground. This is especially true if we consider that most of the occulted stars observed up to now have magnitudes $V \sim 9-12$ mag, a range in which the Gaia position accuracy is very high.

More precisely, the uncertainty of asteroid astrometry as derived by occultations is due to:

- Timing uncertainty and timing errors. Starting from the 90s electronic timing synced to GPS has been increasingly adopted, but older events were sometimes timed by eye and manual chronometer, thus inducing a potentially larger uncertainty subject to personal equations. Occasional errors have been detected also on GPS timings. When no bias is introduced by technical issues, the uncertainty is essentially dominated by the duration of the single exposures used to sample the stellar flux. The timing error is translated to an uncertainty on the position of the asteroid along the direction of its apparent motion.

- The uncertainty of the relative star-asteroid distance transverse to apparent motion, at point of closest appulse. This uncertainty can vary in a wide range, from the order of the apparent asteroid size (when only one occultation chord is observed) to a very small fraction of it (when several occultation chords are measured and the asteroid profile is well resolved).

- The limited accuracy on the position of the star. This is strictly dependent on the properties of the stellar catalogue used to reduce the observation, and it is the main factor impacted by the GDR1/TGAS release.

The occultation database provides errors in both timings and transverse direction (the first two components above). Concerning timings, a large range of uncertainty values is present, but a clear peak around $\sim 0.05 \mathrm{~s}$ appears. For a typical main belt apparent motion of 10-15 mas s${ }^{-1}$, this corresponds to an error around 0.5-1 mas.

Concerning transverse errors, the distribution is clearly bimodal. A first peak for small values ( $\sim 1$ mas) corresponds to the best observed, multi-chord events. A second, much more spread-out set of values, has a wide, flat maximum in the range 20-40 mas (see Fig. 5). This is the same order of the typical apparent radius of the occulting asteroids.

For this first attempt of exploitation of occultation astrometry, we consider only the transverse uncertainty, as, in general, it is larger and dominates the error budget. This is not really a limitation at present, as an accurate use of the timing information is much more delicate and could require a detailed check of those occultation events that could be affected by timing anomalies.

By being conservative and using a single uncertainty value, we believe that we incorporate most error sources in our bulk exploitation of occultation data, without optimistic assumptions. In a forthcoming work we will consider a more detailed analysis of selected asteroids and events.

Concerning the occulted stars we apply the following approach:

- We match the position of the star to the GDR1, looking for the position of corresponding sources in a 2 arcsec radius; we then apply a further check on the consistency of the magnitude (which is in fact redundant, as no ambiguities are found). The position of the matched source in GDR1 is adopted for the occultation. For stars not present in GDR1, the corresponding events are discarded.

- If the star is in TGAS, the position is corrected for its proper motion, consistently with the time delay between the observed event and the catalogue epoch.

- If a star is not in TGAS, it does not have a proper motion. In this case we consider the difference in position between the UCAC4 position and GDR1 to compute an approximate proper motion.

This approach has clear limitations for non-TGAS sources (mainly due to the zonal error in UCAC4) and further tests of GDR1 against other astrometric catalogues could provide useful information; we consider that this investigation, however, is beyond our immediate goals of globally testing the approach on the whole set of occultations.

\subsection{Orbit adjustement}

We attempted an orbital determination, using occultation astrometry alone, for all asteroids that have a historical record of more than four occultations. The observation weights are represented by the transverse accuracy, as explained above. Our fitting procedure rejects observations whose residual is incompatible with the weight (Carpino et al. 2003). No other observations are included for the moment in the orbit fitting procedure.

For each orbit we determine the uncertainty on the semimajor axis $\sigma_{\mathrm{a}}$ and use it as an indicator of the orbit accuracy. In Fig. 6 we compare the accuracy of our results, to that obtained from fitting all the available observation from the MPC: the value of $\sigma_{\mathrm{a}}$ is provided by the AstDys ${ }^{5}$ online repository. One should note that this last orbital solution also contains the contributions of stellar occultations. However, as up to now no case-by-case study of occultation astrometry has been carried out, all such measurements were given a weight of 200 mas (Farnocchia et al. 2015a). Such a weight, coupled to their small number relative to approximately thousands of CCD data points, makes their role negligible in the MPC orbital solution.

We have also computed the orbital uncertainties that could be obtained from the original stellar positions given in the occultation database. These are extracted, case by case, from the best data available, mostly from Tycho/HIPPARCOS, UCAC2, and UCAC4. A comparison of the upper and lower panel of Fig. 6 clearly shows that GDR1 brings an overall improvement by a full order of magnitude.

\footnotetext{
5 http://hamilton.dm . unipi.it/astdys/index $\cdot \mathrm{php}$ ?pc=0
} 

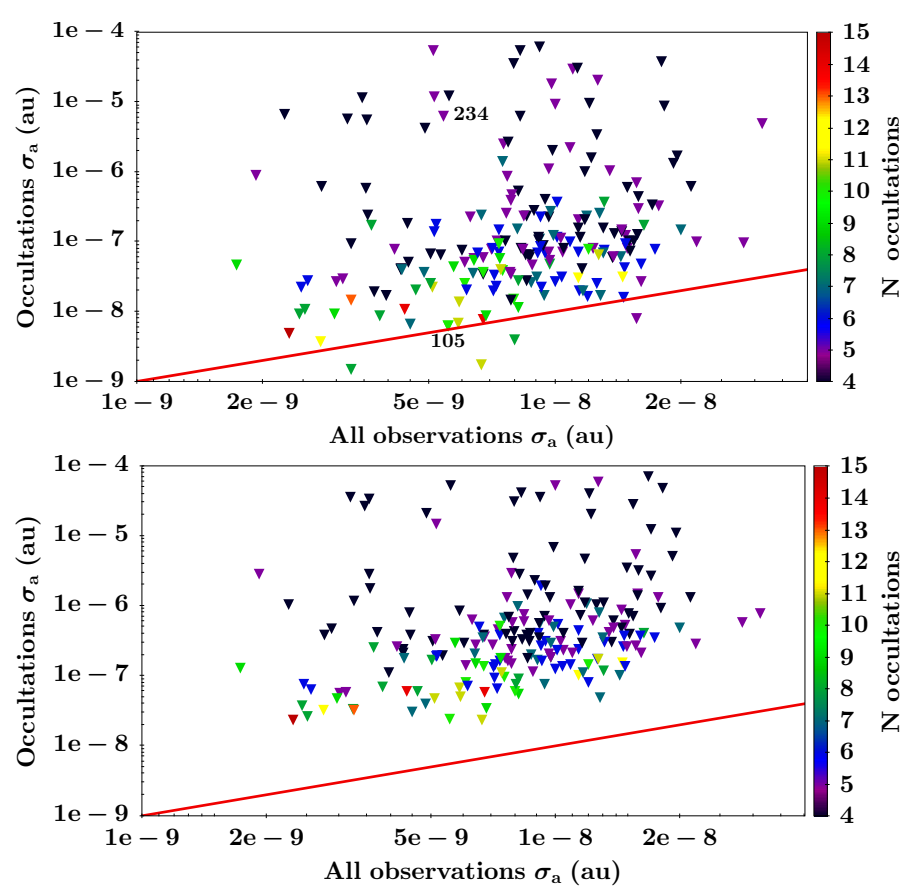

Fig. 6. Upper panel: semi-major axis uncertainties for asteroids with occultations. The values obtained from the use of all observations are on the horizontal axis. On the vertical axis, we show the uncertainty obtained when fitting positions derived from occultation astrometry only, with the procedure described in the text. The line represents equal uncertainties. The symbol colour is related to the number of occultations used. Bottom panel: same as upper panel, but using pre-Gaia astrometry.

Figure 6 shows that properly weighted occultations, even if taken alone, can provide very reasonable orbital solutions for some objects. Of course this is only true for asteroids with a sufficient number of occultations of good quality. All the bad orbital solutions with $\sigma_{a}>10^{-6}$ au have 4-5 astrometric positions from stellar occultations. As a rule of thumb, we can say that above approximately ten astrometric points, uncertainties are not worse than one order of magnitude with respect to the solution obtained with all the observations (thousands of measurements in general).

The performance obtained on a few asteroids, whose orbits show better residuals when only occultations are used, is remarkable. However, for a similar number of occultations, there is a considerable spread in the quality of the solution from one asteroid to the other. For instance, among the best performers, objects with 10 to 15 occultations are found, but also several with only 4 or 5 observed events. For an interpretation of this evidence, both the occultation quality and the distribution of the observed occultations along the asteroid orbit have to be considered.

We illustrate two typical situations in the following. The first one concerns the asteroid (105) Artemis, having ten occultations covering $34 \mathrm{yr}$ and closely matching in performance the accuracy by occultation astrometry and the one with "all data" (1733 astrometric measurements, spanning $112 \mathrm{yr}$ ). The orbit uncertainty is $\sigma_{\mathrm{a}} \sim 4 \times 10^{-9}$ au. An inspection of the residuals of the orbit fitted to occultation astrometry (Fig. 7, upper panel) shows that few multi-chord events are clustered around residuals smaller than 10 mas. A couple of observations are rejected, as their residuals are too large ( $\sim 70$ mas) with respect to their weight. This anomaly could be due to specific problems with the occultations (undetected errors in the observation or the interpretation) or
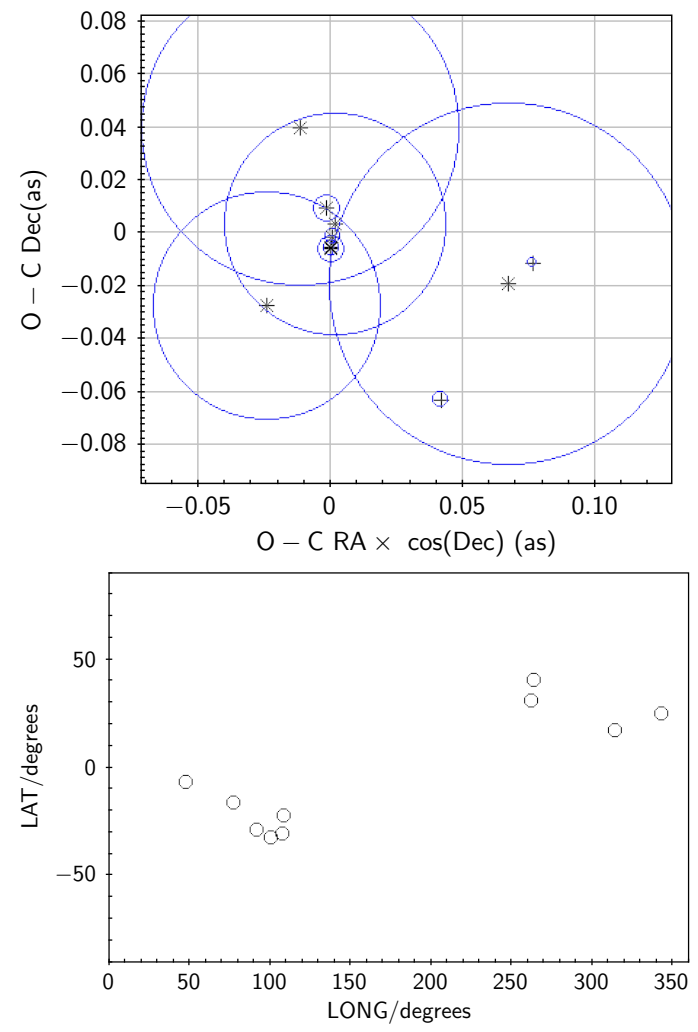

Fig. 7. Upper panel: residuals, with respect to the orbit computed from occultations, for the astrometry of (105) Artemis. Each symbol represents a single occultation event. Observations that are used for the final orbital fit are marked with stars. Crosses correspond to measurements that are automatically rejected. The circles show the nominal uncertainty (weight) of the occultations. Multi-chord events correspond to smaller circles. In the lower panel, the distribution of the occulted stars in ecliptic coordinates is shown.

with the stellar astrometry. Eventually, four events have uncertainties comparable to the apparent radius of the asteroid at the occultation epochs (60-70 mas) and are fully compatible with the solution.

The corresponding distribution of the occulted stars in ecliptic longitude is also shown and covers relatively well about two thirds of the orbit. A good coverage in longitude is certainly required to appropriately constrain the orbit. A counter example is provided by the case of (234) Barbara (Fig. 8). The residuals for the five occultations (covering less than one year) span a smaller range (20 mas) than the case of (105) Artemis (100 mas), and three multi-chord events exhibit residuals better than 2 mas. Yet, the uncertainty for the occultation orbit is poor, around $\sigma_{\mathrm{a}} \sim 5 \times 10^{-5}$ au. This is clearly explained by the fact that the observations are strongly clustered on a very restricted arc of the asteroid orbit, thus preventing any accurate solution.

\section{Conclusions}

We illustrate three situations on which the first Gaia data release, GDR1, has an immediate impact regarding astrometry and orbits of SSOs: the rating of the NEA impact risk, the exploitation of data on very short arcs, and that of stellar occultations. They have each a factor of improvement, with respect to previous catalogues, of about one order of magnitude, corresponding to the ambition of GDR1. 

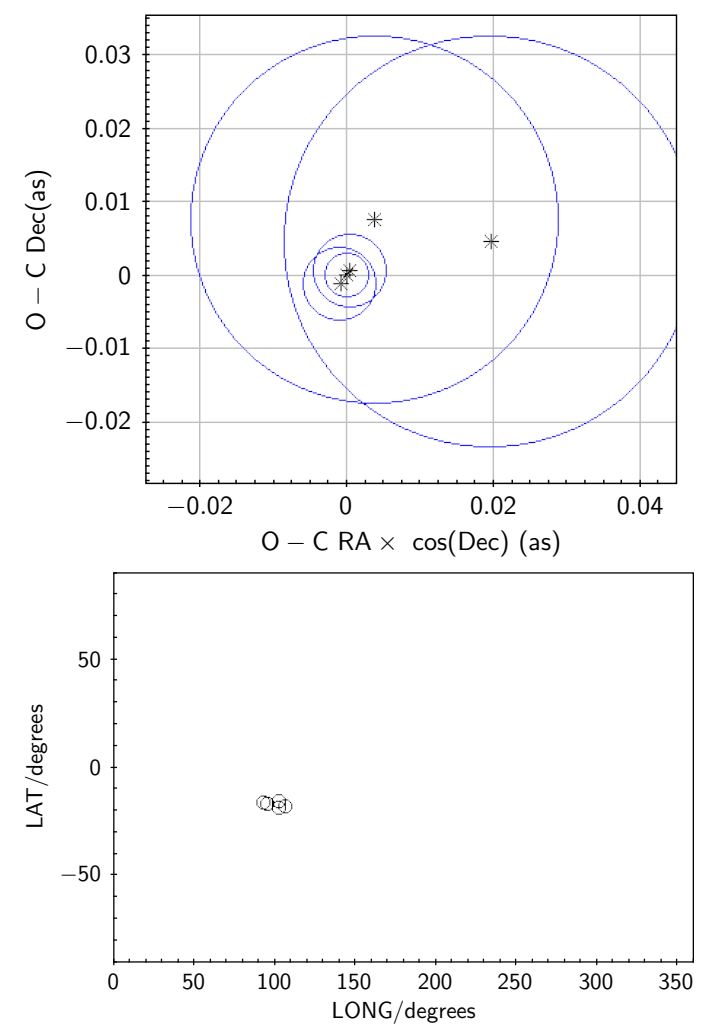

Fig. 8. Same as Fig. 7, for the asteroid (234) Barbara.

To put our results in perspective, we expect that another dramatic improvement will occur with GDR2, that will benefit from a much higher number of observations and more accurate calibrations and will include parallaxes and proper motions for all stars.

We consider that the perspective of exploiting stellar occultations for obtaining precise astrometry is particularly interesting. In fact, in this case the accuracy can be close to that of the star. As we dispose of a complete record of past observations over a few decades, and future observations will be secured in growing numbers, there exists a concrete perspective of expanding the time frame of Gaia-level astrometry beyond the duration of the mission.

Acknowledgements. We thank the referee Dr. Fabrizio Bernardi for his helpful comments, which have improved the quality of the paper. This work has made use of data from the European Space Agency (ESA) mission Gaia (http://www. cosmos.esa.int/gaia), processed by the Gaia Data Processing and Analysis Consortium (DPAC, http://www.cosmos.esa.int/web/ gaia/dpac/consortium). Funding for the DPAC has been provided by national institutions, in particular the institutions participating in the Gaia Multilateral Agreement.

\section{References}

Altmann, M., Bouquillon, S., Taris, F., et al. 2014, in Observatory Operations: Strategies, Processes, and Systems V, Proc. SPIE, 9149, 91490P

Bouquillon, S., Barache, C., Carlucci, T., et al. 2014, in Software and Cyberinfrastructure for Astronomy III, Proc. SPIE, 9152, 915203

Bouquillon, S., Mendez, R. A., Altmann, M., et al. 2017, A\&A, 606, A27

Carpino, M., Milani, A., \& Chesley, S. R. 2003, Icarus, 166, 248

Chesley, S. R., Baer, J., \& Monet, D. G. 2010, Icarus, 210, 158

Desmars, J., Bancelin, D., Hestroffer, D., \& Thuillot, W. 2013, A\&A, 554, A32

Dunham, D. W., Herald, D., Frappa, E., et al. 2016, NASA Planetary Data System, 243

Farnocchia, D., Chesley, S. R., Chamberlin, A. B., \& Tholen, D. J. 2015a, Icarus, 245, 94

Farnocchia, D., Chesley, S. R., \& Micheli, M. 2015b, Icarus, 258, 18

Gaia Collaboration (Brown, A. G. A., et al.) 2016, A\&A, 595, A2

Liebe, C. C. 1995, IEEE Aerospace and Electronic Systems Magazine, 10, 10

Lindegren, L., Lammers, U., Bastian, U., et al. 2016, A\&A, 595, A4

Milani, A., Gronchi, G. F., Vitturi, M. D., \& Knežević, Z. 2004, Mech. Dyn. Astron., 90, 57

Milani, A., Chesley, S. R., Sansaturio, M. E., Tommei, G., \& Valsecchi, G. B. 2005a, Icarus, 173, 362

Milani, A., Sansaturio, M. E., Tommei, G., Arratia, O., \& Chesley, S. R. 2005b, A\&A, 431, 729

Roeser, S., Demleitner, M., \& Schilbach, E. 2010, AJ, 139, 2440

Spoto, F., Milani, A., Tommei, G., et al. 2017, Icarus, submitted

Tanga, P., \& Delbo, M. 2007, A\&A, 474, 1015

Tanga, P., Hestroffer, D., Delbò, M., et al. 2008, Planet. Sp. Sci., 56, 1812 